\title{
In search of the golden ratio for cannabis allergy: utility of specific allergen-to-total IgE ratios
}

\author{
Ine Decuyper ${ }^{1}$, Hans-Peter Rihs ${ }^{2}$, Christel Mertens ${ }^{1}$, Athina Van Gasse ${ }^{1}$, Jessy Elst ${ }^{1}$, \\ Margaretha Faber ${ }^{1}$, Margo Hagendorens ${ }^{1}$, Vito Sabato ${ }^{1}$, and Didier Ebo ${ }^{3}$ \\ ${ }^{1}$ Universiteit Antwerpen Faculteit geneeskunde en gezondheidswetenschappen \\ ${ }^{2}$ Institute for Prevention and Occupational Medicine of the German Social Accident \\ Insurance, Institute of the Ruhr-Universität Bochum, IPA \\ ${ }^{3}$ University of Antwerp Faculty of Medicine and Health Sciences
}

June 1, 2021

\section{ABBREVIATIONS}

CA cannabis allergy

CCDs cross-reactive carbohydrate determinants

CI confidence interval

CSA cannabis sativa allergic patients

nsLTP s nonspecific lipid transfer proteins

P+LTP- controls sensitized to pollen without nsLTP sensitization

$\mathrm{P}+\mathrm{LTP}+$ controls sensitized to pollen and nsLTPs

(r) recombinant

sIgE specific immunoglobulin E

ST skin tests

tIgE total immunoglobulin E

KEYWORDS

Cannabis allergy, sIgE, total IgE, allergy diagnosis, sIgE-to-total IgE ratio

To the Editor,

The most important "diagnostic test" for CA is a detailed history. However, a positive history is no absolute proof of CA, mainly because of physiological effects of cannabis i.e. (rhino)conjunctivitis presence and possibly because of incorrect interpretation or recollection of symptoms by the patients under the drug's influence. Consequently, clinical suspicion of CA requires confirmatory testing. A cannabis challenge, being excluded for obvious ethical/legal reasons, documentation of CA generally starts with skin testing (ST) or specific IgE-quantification. However, in the absence of a standardized extract for ST, many will use prickprick tests (e.g., with buds, leaves, seeds) ${ }^{12}$. Crude plant parts or extracts thereof, can contain both genuine and cross-reactive allergenic components. Consequently, positive results of crude extract ST and sIgE should always be interpreted cautiously, as it might merely reflect (cross-) sensitization instead of allergy. 
Here, we sought to investigate whether serological diagnosis of CA could benefit from an adjustment for tIgE. For this purpose, sIgE-to-tIgE ratios were calculated for sIgE hemp (FEIA, ImmunoCAP ThermoFisher Scientific), sIgE to recombinant (r) Can s 3 and rCan s 5 (Cytometric Bead Assay) as detailed elsewhere ${ }^{3}$. A sIgE rCan s 3-to-rPru p 3 and sIgE rCan s 5-to-rBet v 1 ratio was also calculated. Negative sIgE values were excluded, as these cannot benefit from such an adjustment. Patients were selected from our previously published data ${ }^{4}$; cannabis sativa allergic patients (CSA), controls with a pollen but no nsLTP sensitization (P+LTP-) and controls with both pollen and nsLTP sensitizations $(\mathrm{P}+\mathrm{LTP}+)$ were enrolled.

Detailed demographic information is shown in table 1 of our previously published article ${ }^{3}$. As shown in figure 1, the distribution of the sIgE hemp-to-tIgE ratio and $\operatorname{sIgE}$ rCan s 5-to-tIgE differed significantly between controls and CSA. Using a cut-off of 0.02 for sIgE hemp-to-tIgE, it was shown that a specificity of $93 \%$ (95\% confidence interval (CI), 85-98\%) could be reached (table 1). A second, lower cut-off (0.005) can benefit most of the CSA population (sensitivity $77 \%$ (95\% CI 67-85\%)) and retains a relatively good specificity $79 \%$ (95\% CI $68-87 \%)$.

For sIgE rCan s 5-to-tIgE, a cut-off of 0.01 could possibly be beneficial. However, small group numbers make it difficult to estimate its true value. The ratio of sIgE rCan s 3 -to-tIgE did not show added value ( $\mathrm{p}=0.86)$.

Comparing CSA patients with and without anaphylaxis; no added value was found to identify (a risk of) cannabis related anaphylaxis for any of the explored sIgE-to-tIgE ratios (sIgE hemp-to-tIgE- $>$ p=0.104; $\operatorname{sIgE}$ rCan s 3-to-tIgE- $>$ p=0.416; sIgE rCan s 5-to-tIgE- $>$ p=0.84, data not shown).

Finally, ratios of similar protein families i.e. sIgE rCan s 3-to-sIgE rPru p 3 and sIgE rCan s 5-to-sIgE rBet v 1 were explored, showing no additional diagnostic value (see figure E1 in the online repository).

These results indicate that sIgE-to-tIgE ratios might have a place in the diagnostic approach of CA. In the case of a definite history of cannabis related symptoms we recommend (figure E2) a sIgE hemp assay (sensitivity $82 \%$ (95\% CI 74-89\%) and specificity $32 \%(95 \%$ CI $20-45 \%))^{3}$. A negative result significantly reduces the chance of IgE-mediated CA. A positive result should be followed by a sIgE hemp-to-tIgE ratio as it notably increases test specificity. Where available, it is worthwhile using cannabis component resolved diagnostics as it was shown that over two-thirds of CSA who experienced anaphylaxis are Can s 3 sensitized ${ }^{3}$. This study is bound by certain limitations; group numbers vary between the different analyses because of insufficient patients' sera. Additionally, we could not explore sIgE rCan s 2-to-tIgE, sIgE rCan s 4-to-tIgE and sIgE rCan s 4-to-sIgE rBet v 2, because of insufficient group numbers. Finally, there is no guarantee that these results can be extrapolated to other CSA populations or different geographical regions. The results of these study are of a preliminary nature. Repetition of our methods in other, ideally larger populations are feasible to see whether these results can be confirmed and remain intact in different CA populations.

\section{FIGURES \& TABLES}

FIGURE 1

\section{Hosted file}

image1.emf available at https://authorea.com/users/417276/articles/524443-in-search-of-thegolden-ratio-for-cannabis-allergy-utility-of-specific-allergen-to-total-ige-ratios

Figure 1: dotplot with boxplot overlay showing A: sIgE hemp-to-tIgE ratio for controls $(n=77)$ and CSA ( $n=94)$. B: sIgE rCan s 3-to-tIgE for controls ( $n=21)$ and CSA ( $n=55)$. Green $=P+L T P-$, blue $=P+L T P+$, orange $=C S A$-no anaphylaxis, red=CSA-anaphylaxis. C: sIgE $r C a n$ s 5-to-tIgE ratio for controls $(n=18)$ and CSA $(n=17) .{ }^{*}$ Comparison of controls and CSA was performed by Mann-Whitney U testing

TABLE 1

\begin{tabular}{lllll}
\hline & sIgE hemp-to-tIgE & sIgE hemp-to-tIgE & sIgE hemp-to-tIgE & sIgE hemp-to-tIgE \\
\hline & $<\mathbf{0 . 0 2}$ & {$[\mathbf{?}] \mathbf{0 . 0 2}$} & $<\mathbf{0 . 0 0 5}$ & {$[?] 0.005$} \\
Controls & 70 & 5 & 59 & 16
\end{tabular}




\begin{tabular}{lllll}
\hline & sIgE hemp-to-tIgE & sIgE hemp-to-tIgE & sIgE hemp-to-tIgE & sIgE hemp-to-tIgE \\
\hline CSA & 58 & 34 & 21 & 71 \\
Sensitivity (95\%CI*) & $37 \%(27-48 \%)$ & $37 \%(27-48 \%)$ & $77 \%(67-85 \%)$ & $77 \%(67-85 \%)$ \\
Specificity (95\%CI*) & $93 \%(85-98 \%)$ & $93 \%(85-98 \%)$ & $79 \%(68-87 \%)$ & $79 \%(68-87 \%)$ \\
Pearson Chi-square & $\mathrm{p}<0.01$ & $\mathrm{p}<0.01$ & $\mathrm{p}<0.01$ & $\mathrm{p}<0.01$ \\
\hline
\end{tabular}

* Confidence interval

REPOSITORY MATERIAL

FIGURE E1

\section{Hosted file}

image2.emf available at https://authorea.com/users/417276/articles/524443-in-search-of-thegolden-ratio-for-cannabis-allergy-utility-of-specific-allergen-to-total-ige-ratios

Figure E1: dotplot with boxplot overlay. sIgE rCan s 3-to-sIgE rPru p3 for controls ( $n=20)$ and CSA ( $n=53)$. sIgE rCan s 5-to-sIgE rBet $v 1$ for controls $(n=18)$ and $C S A(n=18) . \quad$ Green $=P+L T P-, b l u e=P+L T P+$, orange $=C S A$-no anaphylaxis, red=CSA-anaphylaxis. ${ }^{*}$ Comparison of controls and CSA was performed by a Mann-Whitney U test.

FIGURE E2

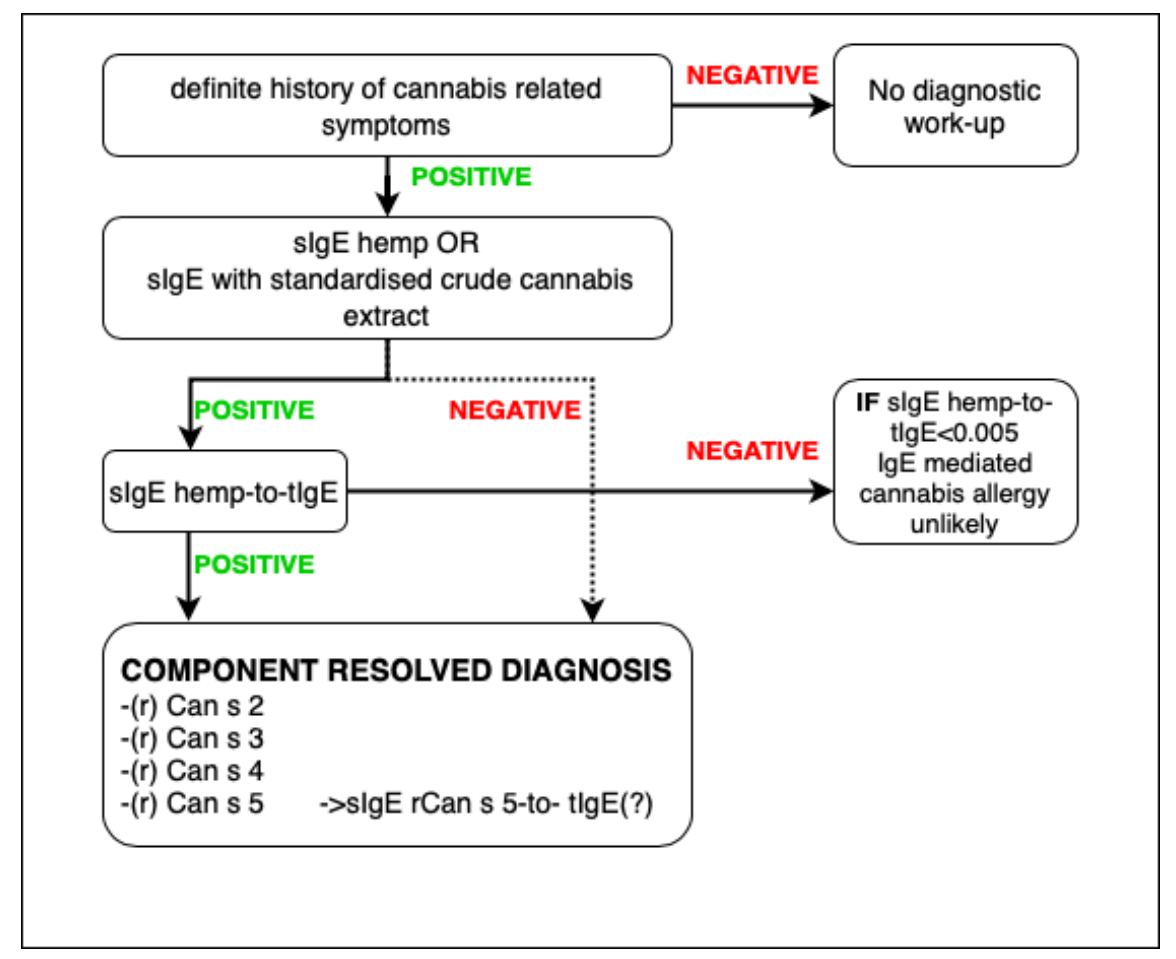

Figure E2: Proposed algorithm for serological diagnosis of IgE mediated cannabis sativa allergy. Performance of component resolved diagnosis based on availability.

REFERENCES 
1. Tessmer A, Berlin N, Sussman G, et al. Hypersensitivity reactions to marijuana. Ann Allergy Asthma Immunol 2012;108(4):282-4. doi: 10.1016/j.anai.2012.01.008 [published Online First: 2012/04/04]

2. Nayak AP, Green BJ, Sussman G, et al. Characterization of Cannabis sativa allergens. Ann Allergy Asthma Immunol 2013;111(1):32-7. doi: 10.1016/j.anai.2013.04.018 [published Online First: 2013/06/29]

3. Decuyper, II, Van Gasse AL, Faber MA, et al. Exploring the Diagnosis and Profile of Cannabis Allergy. J Allergy Clin Immunol Pract2019;7(3):983-89 e5. doi: 10.1016/j.jaip.2018.09.017 [published Online First: 2018/10/03]

4. Ebo DG, Decuyper, II, Rihs HP, et al. IgE-binding and mast cell activating capacity of the homologue of the major birch pollen allergen and profilin from Cannabis sativa. J Allergy Clin Immunol Pract2021 doi: 10.1016/j.jaip.2021.02.012 [published Online First: 2021/02/20]

Ine I. Decuyper, $\mathrm{MD}, \mathrm{PhD}^{1,2}$, Hans-Peter Rihs, $\mathrm{PhD}^{3}$, Christel H. Mertens, MLT ${ }^{1}$, Athina Ludovica Van Gasse, MD, $\mathrm{PhD}^{1,2}$, J. Elst, MSc ${ }^{1}$, Margaretha A. Faber, MD, $\mathrm{PhD}^{1}$, Margo M. Hagendorens, MD, $\mathrm{PhD}^{1,2}$, Vito Sabato, $\mathrm{MD}, \mathrm{PhD}^{1,4}$, and Didier G. Ebo, $\mathrm{MD}, \mathrm{PhD}^{1,4}$

${ }^{1}$ University of Antwerp, Faculty of Medicine and Health Sciences, Department of Immunology, Allergology, Rheumatology and the Infla-Med Centre of Excellence, Antwerp (Belgium) and Immunology, Allergology, Rheumatology, Antwerp University Hospital, Antwerp (Belgium)

${ }^{2}$ University of Antwerp, Faculty of Medicine and Health Sciences, Department of Paediatrics and the InflaMed Centre of Excellence, Antwerp (Belgium) and Paediatrics, Antwerp University Hospital, Antwerp (Belgium)

${ }^{3}$ Institute for Prevention and Occupational Medicine of the German Social Accident Insurance (IPA), Institute of the Ruhr-University Bochum, Bochum, Germany

4 AZ Jan Palfijn Gent, Department of Immunology and Allergology, Ghent, Belgium

Corresponding author:

Prof. dr. Didier G. Ebo

Laboratory of Immunology

Campus Drie Eiken T5.95

Universiteitsplein 1

2610 Antwerpen

$++3232652595$

immuno@uantwerpen.be

ORCID ID:

Ine Decuyper: https://orcid.org/0000-0001-8127-5791

Hans-Peter Rihs: https://orcid.org/0000-0002-8991-1526

Christel Mertens: https://orcid.org/0000-0003-2359-0771

Athina Van Gasse: https://orcid.org/0000-0003-1657-5135

Jessy Elst: https://orcid.org/0000-0003-3506-8200

Margaretha Faber: https://orcid.org/0000-0002-1277-5052

Margo Hagendorens: https://orcid.org/0000-0001-6361-9503

Vito Sabato: https://orcid.org/0000-0002-1321-314X 
Didier Ebo: https://orcid.org/0000-0003-0672-7529

Contributions statement :

Ine I. Decuyper participated in interpretation of data, literature search, data analysis, writing the manuscript; Hans-Peter Rihs participated in discussion diagnostic techniques and proofreading final text; Jessy Elst participated in proofreading final text; Athina L. Van Gasse participated in proofreading final text; Vito Sabato participated in proofreading final text; Margaretha A. Faber participated in literature search and proofreading final text; Christel Mertens participated in performing $\operatorname{IgE}$ quantifications, interpretation of diagnostic data and proofreading final text; Margo M. Hagendorens participated in proofreading final text; Didier G. Ebo participated in litera- ture search, data analysis, writing and proofreading final text.

Funding sources: This work was supported by the Agency for Innovation by Science and Technology (IWT) [grant number 140185]; ALVG is a fellow of the FWO [1113617N]. The authors thank B. Van Camp, head of the Unit 'drug production', Central Drug Department, Directorate of organised crime, Belgian Federal Judicial police for providing Cannabis sativa plants. DGE is a Senior Clinical Researcher of the FWO $(1800614 \mathrm{~N})$.

Conflicts of interest: None of the authors state a conflict of interest for this work. 